\title{
Water drops passing through a forest fuel bed without its burning and during its thermal decomposition
}

\author{
Roman S. Volkov, Alyona O. Zhdanova*, and Victoria A. Kirienko \\ National Research Tomsk Polytechnic University, 634050 Tomsk, Russia
}

\begin{abstract}
This article contains the findings of experimental research into determination of the liquid volume passed through forest fuel bed (pine needles) without burning and during its thermal decomposition. The experiments were carried out using hollow cylinders with fixed geometric parameters (height $h_{\mathrm{f}}$ and diameter $d_{\mathrm{f}}$ ) for creating model of fire sources. When analyzing the results we determined the dependences of the drops mass after the passage of the forest fuel bed as a function of the initial mass. All necessary conclusions about the way drop volume effects on the mass loss after passing through a forest fuel bed are included.
\end{abstract}

\section{Introduction}

The problem of the emergence, monitoring and prevention of forest fires has been urgent for a long time. The total area of the Earth covered with forest is about 3866 million hectares. It occupies about one third of the land surface. Forest ecosystems are suppliers of ecological resources for all living things and sources of economically valuable products [1]. According to statistics, a forest fire is registered on the planet every 5 seconds. The average annual number of fires reaches 5.5 million [2]. Doubtless, such number of fires brings with it cost-intensive losses: human, economic, reduction of forest resources, destruction of biogeocenoses, etc. For example, according to Russian statistics, forest fires destroyed 70 million cubic meters of wood (2002), about 700 thousand hectares of forest plantations (2000), 800 thousand hectares of forests (2009). In fact, the real damage from forest fires is much higher than published in documents [3, 4]. Despite huge losses, Russian forests are still very important. Furthermore, forests have economic and global ecological significance. Therefore, the forest fires problem is under steadfast attention

The solution of forest fires problem requires different kinds of activities. It includes the prediction of fires, monitoring and control of areas under the threat, development and application of an effective extinguishing system. All aspects are developing and progressing at the moment. Many groups of scientists are working on new methods of firefighting. So, in modern realities, there are two methods of fire-extinguishing. According to the first method, firefighters should act on the fire edge (active methods). This method includes such technologies as fire beating, blowing, throwing the soil on the fire edge, quenching with water or chemicals. The second method involves creating mineralized barrier bars or ditches, as well as annealing (passive methods) [5].

\footnotetext{
Corresponding author: zhdanovaao@tpu.ru
} 
The most common method of extinguishing fires in Russia is quenching using water with aviation help. However, such technology entails irrational consumption of huge amounts of water or other quenching liquids. Therefore, various studies are being conducted in order to find ways of reducing liquid losses. Some necessary characteristics have been already determined. These include droplet sizes, thickness of the liquid film layer [6], and necessary droplets position in the water shell [7].

During carrying out such studies and using its results, it is necessary to take into account the fact that part of the extinguishing liquid will not take part in the fire extinguishing process. This fact needs to be studied in details

The purpose of this research was to estimate the amount of liquid that flows into the ground and does not participate in suppressing the thermal decomposition process.

\section{Experimental setup and procedure}

Fig.1 shows the scheme of experimental setup used in carrying out researches. This setup provides registration of a forest fuel bed temperature in three points of standardized fire. Also, the setup enable us to fix the forest fuel (FF) sample weight as well as droplet masses after passing through the dry layer and through the layer during its destruction.

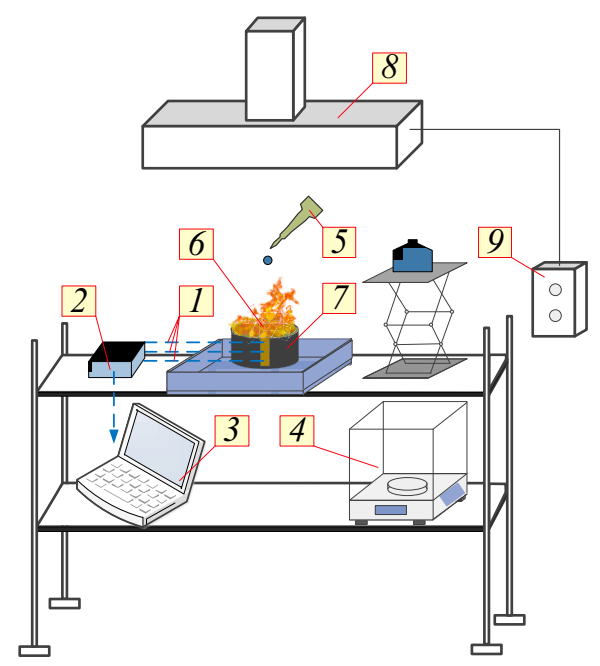

Fig. 1. A scheme of experimental setup: 1 -thermocouples; 2 - high-speed analog input module; $3-$ workstation (PC); 4 - analytical microbalance; 5 - dispenser; 6 - FF; 7 - cylinder with a FF sample; 8 - ventilation system; 9 - control panel of turning on/off ventilation system.

Experimental setup consisted of a cylinder 7 with an FF sample 6 and three type K thermocouples 1 that recorded and transmitted temperature values to workstation 3 through the high-speed analog input module 2. The droplet sizes were regulated by the dispenser (dosing device) 5. The liquid mass that did not participate in suppressing the thermal decomposition process was recorded with laboratory microbalance 4.

Spruce needles were chosen as a forest fuel for research. Such choice of material can be explained by structure and porosity of this FF. Using spruce needle makes it possible to register the process of water passing through the bed. For other forest fuels it is difficult to obtain such results. For example, for birch leaves, it is difficult to trace the motion of a drop across the entire layer's thickness. This phenomenon is conditioned by the structure of this forest fuel. A drop can "settle" on a birch leaf and further recording of its motion becomes impossible. The samples used for research was pre-dried for 3-5 days at a temperature of 
about $300 \mathrm{~K}$. Immediately before the experiments we determined FF moisture using thermal drying method. The relative spruce needles moisture content $\gamma_{\mathrm{f}}$ was $7-10 \%$.

Before each experiment, the forest fuel was weighed on an analytical microbalance 4 in order to determine mass $m_{\mathrm{f} 0}$. After that, forest fuel was placed to the bottom of the cylinder 7. The density of samples in each experiment remained constant.

Cylinder made of corrugated aluminum (see fig. 2) was used for create model of fire source. In our experiments cylinder had the diameter $d_{\mathrm{f}}=60 \mathrm{~mm}$ and the height $h_{\mathrm{f}}=40 \mathrm{~mm}$. The model of fire source 2 was placed on a specialized woven surface made of annealed wire of standard quality (GOST 3282-74). The size of the grid cells was $1 \mathrm{x} 1 \mathrm{~mm}$. Below the grid 3 was a drop trap 4 . A vertical hole ( $3 \mathrm{~mm}$ in wide) was made in the cylinder to visually monitor the thermal decomposition of forest fuel (see Fig. 1).

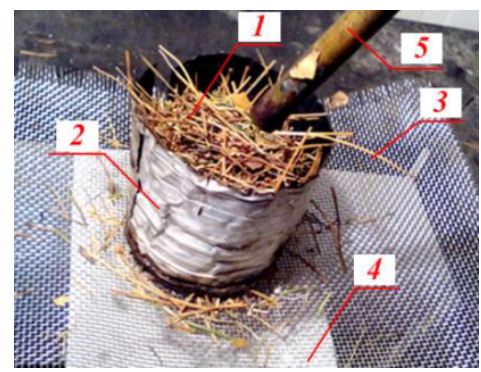

Fig. 2. The video record of the experimental determination of the water mass during passing through FF bed: 1 - spruce needles, 2 - cylinder, 3 - stainless steel grid (tissue type, made of the metal), 4 trap, 5 - dispenser.

Water droplets volume was varied in the range $86-900 \mu \mathrm{L}$ during the series of experiments. The volumes and radii of drops used for the experiments are given in table 1. We conducted from 15 to 20 experiments for each droplet size. Then, we sampled the experimental values of $m_{\mathrm{de}}$ differing by less than $5 \%$ for identical experimental conditions. The process of the experiment was conducted in two stages. The drop was generated by the dispenser 5. After passing through the FF bed, drop fell into the trap 4 (Fig. 2). At the first stage droplet mass after passing through the forest fuel bed to ignition was recorded. The estimation of the water mass, which lingers on the grid 3, was determined. Before starting the experiment, water droplets of considered size were passed through the grid. We measured part of mass that passed from $m_{\mathrm{d} 0}$ to the trap. The average mass loss through the grid surface was $22-24 \%$ of the initial mass $m_{\mathrm{f} 0}$.

Table 1. The volumes and radii of water droplets used in the experiments.

\begin{tabular}{|c|c|c|c|c|}
\hline$V_{\mathrm{d}}, \mu \mathrm{L}$ & 86 & 334 & 776 & 900 \\
\hline$R_{\mathrm{d}}, \mathrm{mm}$ & 2.7 & 4.3 & 5.7 & 5.9 \\
\hline
\end{tabular}

During the second stage of experiment, the cylinder 7 was placed on a stainless steel grid 4 (Fig. 2). Then we set three type-K needle thermocouples on cylinder's symmetry axis (temperature measurement range $223-1473 \mathrm{~K}$, accuracy $\pm 3 \mathrm{~K}$, heat retention no more than $0.1 \mathrm{~s})$. After that the model of fire source was ignited. The criterion for stable FF burning was the excess of FF temperature the thermal decomposition temperature $\left(T_{\text {if }}\right)-370 \mathrm{~K}$. Further, after the readings of all thermocouples reached the value $T_{\text {if }}$, single droplets were fed to the standardized fire. At the last stage, the initial drop mass $m_{\mathrm{d} 0}$ was determined 
using analytical microbalance 4 . We carried out series of experiments for 4 different droplet sizes.

\section{Results and discussion}

During experimental investigations the dependences of the droplets' mass loss on their initial masses during passage through the FF bed without combustion and in the combustion process were obtained (Fig.3). The curves were built for drops with size $R_{\mathrm{d}} \approx 4.3$ $\mathrm{mm}, R_{\mathrm{d}} \approx 5.7 \mathrm{~mm}$ и $R_{\mathrm{d}} \approx 5.9 \mathrm{~mm}$. Weight loss value was calculated by formula

$$
\Delta m=m_{d 0}-m_{d e}
$$

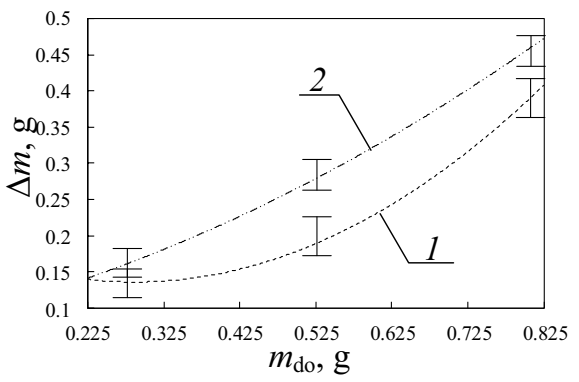

Fig. 3 Dependence of the parameter $\Delta \mathrm{m}$ on the initial mass of droplets $m_{\mathrm{d} 0},(\Delta \mathrm{m}$ is the parameter characterizing the mass changing during the passage through forest fuel bed, taking into account the losses on the grid): 1 - water drops passing through FF bed without its burning; 2 - water drops passing through FF bed during its thermal decomposition.

The obtained results showed that droplets with a radius $R_{\mathrm{d}}=2.7 \mathrm{~mm}$ completely "settled" in FF bed (without and during its burning). Therefore, dependency curves were built for droplets with other dimensions. For them complete subsidence in the bed did not occur. Analysis of the dependencies presented in figure 3 allows us to conclude that the more droplet size the greater mass fraction it loses during the passing through the bed. This fact can be explained by large contact area with FF fragments for drops with volume $V_{\mathrm{d}} \approx 900 \mu \mathrm{L}$.

Furthermore, during the analysis of the obtained experimental data, dependence of the droplets masses $\left(R_{\mathrm{d}} \approx 4.3 \mathrm{~mm}, R_{\mathrm{d}} \approx 5.7 \mathrm{~mm}\right.$ and $\left.R_{\mathrm{d}} \approx 5.9 \mathrm{~mm}\right)$ after passing through the $\mathrm{FF}$ bed on the water mass that has passed though the grid was built (fig.4).

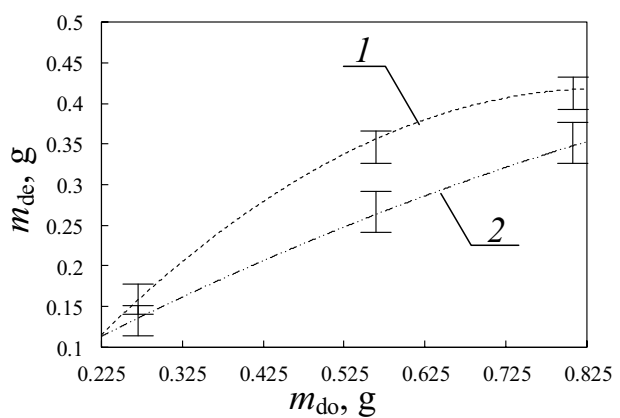

Fig.4 Dependence of the droplets masses $\left(R_{d} \approx 4.3 \mathrm{~mm}, R_{d} \approx 5.7 \mathrm{~mm}\right.$ and $\left.R_{d} \approx 5.9 \mathrm{~mm}\right)$ after passing through FF bed on the water mass that has passed though the grid: 1 - water drops passing through FF bed without its burning; 2 - water drops passing through FF bed during its thermal decomposition. 
Analysis of the obtained dependences, enable us to make a conclusion about almost linear dependence between masses in case of thermal decomposition process and an increasing dependence during water drops passing through a dry forest fuel. The results of the series of experiments indicate that the most rational way of firefighting is using medium-sized drops of quenching liquid. In such case liquid can reach all FF bed and affect the process of suppressing the decomposition.

Thus, obtained during experiments dependencies can describe the process of liquid drops' mass loss during passing through a FF bed without and during its burning, taking into account the droplet size. The results of experimental studies can be used to estimate liquid volume, which is necessary to extinguish a forest fire.

\section{Conclusion}

As a result of the experimental studies, we established the dependencies of droplets mass loss on the initial one when passing through a forest fuel bed as well as dependencies of droplets mass loss on drops volumes. The obtained results allowed us to determine the range of droplets sizes for spraying in the stream. It will help to minimize the amount of liquid, which is not involved in suppressing the thermal decomposition process. Our conclusions and dependencies should be taken into account when creating numerical models for finding the necessary quenching liquid volume, drops size and times of extinguishing forest fires.

The investigations of heat and mass transfer processes in the suppression of forest fire were supported by Russian Science Foundation (project 14-39-00003). Experiments to determine the specific differences between the processes of thermal decomposition and combustion of various types of forest fuel were carried out within the framework of the President of the Russian Federation grant MK-1684.2017.8.

\section{References}

1. Yu. L. Vorobiev, V. A. Akimov, Yu. I. Sokolov, Forest fires on the territory of Russia: state and problems (Deks-Press, 2004)

2. A. I. Bobko, Fires as geoecological danger, a collection of the conference proceedings "Geography and Geoecology in the Service of Science and Innovative Education”, 319 (2014)

3. L. M. Ivanova, North and the market: the formation of the economic order, 1, 103 (2011)

4. A. V. Konstantinov, V. V. Morkovina, Actual directions of scientific research of the XXI century: theory and practice, 4, 319 (2016)

5. D. S. Stupnikov, Actual directions of scientific research of the XXI century: theory and practice, 3, 201 (2015)

6. A. O. Zhdanova, G. V. Kuznetsov, P. A. Strizhak, Chemical Physics and Mesoscopy, 17, 172 (2015)

7. A. O. Zhdanova, G. V. Kuznetsov, P. A. Strizhak, Chemical Physics and Mesoscopy, 16, 215 (2014) 